Pacific Journal of Mathematic 


\title{
ON PROJECTIONS OF REAL ALGEBRAIC VARIETIES
}

\author{
C. Andradas and J. M. Gamboa
}

In this paper we generalize an earlier result of the authors, showing that any closed semialgebraic set whose Zariski-closure is irreducible, is the projection under a finite map of an irreducible real algebraic set (see Theorem 3.2 below).

1. Introduction. This result is, somehow, striking and shows the wild nature of irreducible real algebraic sets; no matter how complicated (in terms of connected components, pieces of different dimensions, etc...) a closed semialgebraic set is (as far as its Zariski-closure is irreducible, which is a fairly weak condition), there exists an irreducible algebraic set projecting onto it. In this way, some examples of "exotic" algebraic sets are obtained in $\S 4$.

Also in $\$ 4$, as an application, is stated a result on the Harrison's topology of the space of orders of function fields, which in fact was the starting point of this work. The problem is: Is any clopen (i.e. closed and open) subset of the space of orders of a function field the image of the space of orders of a finite extension? This question is proposed in [E-L-W]. The answer is affirmative (see 4.1 below) but as it is usual dealing with orders in function fields we had to translate the problem to geometry using the, well known at this moment, correspondence between clopens and semialgebraic sets of a model of the function field. In particular some results of [D-R] are needed. Thus the question follows as a corollary of the geometric result 3.2.

Throughout the paper $R$ denotes an arbitrary real closed field. Given any real algebraic set $V$ we denote by $V_{c}$ the set of central points of $V$, that is the closure of the regular points of $V$. We work always with the order topology of $R^{n}$. Finally the symbol $\pi$ always stands for the projection into the $n$ first coordinates.

2. The key proposition. Here $V$ will be an irreducible algebraic subset of $R^{n}$. We shall denote by $\mathfrak{p}$ its ideal polynomials and by $R[V]$ its coordinate ring. 
2.1. Proposition. Let $f_{1}, \ldots, f_{p}$ be polynomials in $R\left[X_{1}, \ldots, X_{n}\right]$. Assume that the semialgebraic set $S=V \cap\left(\left\{f_{1} \geq 0\right\} \cup \cdots \cup\left\{f_{p} \geq 0\right\}\right)$ is Zariski-dense in $V$. Then there exists an irreducible algebraic set $W \subset R^{n+1}$ such that $\pi: W \rightarrow V$ is finite and $\pi(W)=S$.

Proof. The proof follows closely the one of the analogous result in the case $V=R^{n}$ which appears in [A-G], Proposition 2.1. So, there we proved that for any $\lambda_{1}, \lambda_{2} \in R, 0<\lambda_{2}<\lambda_{1}$, the set of zeros $\tilde{W} \subset R^{n+1}$ of the polynomial $F(T, \underline{X}) \in R\left[X_{1}, \ldots, X_{n}, T\right]$,

$$
\begin{aligned}
F(T, X)= & Q f_{p}\left(T^{2}-\lambda_{2} f_{1}\right)-Q T^{2}\left(T^{2}-\lambda_{1} f_{1}\right) \\
& -\left(T^{2}-\lambda_{2} f_{1}\right) \sum_{i=2}^{p-1} T^{2}\left(T^{2}-2 f_{i}\right) Q_{i}
\end{aligned}
$$

verifies that $\pi: \tilde{W} \rightarrow R^{n}$ is finite and $\pi(\tilde{W})=\tilde{S}=\left\{f_{1} \geq 0\right\} \cup \cdots \cup$ $\left\{f_{p} \geq 0\right\}$, where it is:

$$
\begin{aligned}
& Q(T, \underline{X})=\prod_{i=2}^{p-1}\left(T^{2}-f_{i}\right), \quad \text { and } \\
& Q_{i}(T, \underline{X})=Q(T, \underline{X}) /\left(T^{2}-f_{l}\right), \quad(i=2, \ldots, p-1) .
\end{aligned}
$$

Now, let $V^{\prime}=V \times R$ be the algebraic set defined by $p R[T, \underline{X}]$. It is obvious that $\pi\left(\tilde{W} \cap V^{\prime}\right)=S$. Then all our work is dedicated to show that for some $\lambda_{1}, \lambda_{2}, \tilde{W} \cap V^{\prime}$ is an irreducible algebraic set. For that we will use Bertini's theorem. First of all we shall assume that $f_{i} \notin \mathfrak{p}$ (all $i=1, \ldots, p)$, since otherwise we would have $S=V$ and then the proposition is trivial. Also, we shall assume $f_{l}-\lambda f_{j} \notin \mathfrak{p}$ for all $i, j \in\{1, \ldots, p\}$, $\lambda \in R$. For if $f_{i}-\lambda f_{j} \in \mathfrak{p}$ then either $\left\{f_{i} \geq 0\right\} \cap V=\left\{f_{j} \geq 0\right\} \cap V$ if $\lambda>0$ and we just omit one of them, or $\left\{f_{l} \geq 0\right\} \cap V=\left\{-f_{j} \geq 0\right\} \cap V$, if $\lambda<0$, what implies again $S=V$. Next, we write $F(T, \underline{X})$ in the form

$$
F(T, \underline{X})=P_{0}+\lambda_{1} P_{1}+\lambda_{2} P_{2}
$$

where

$$
\begin{aligned}
& P_{0}=Q f_{p} T^{2}-Q T^{4}-T^{4} \sum_{i=2}^{p-1}\left(T^{2}-2 f_{i}\right) Q_{i} \\
& P_{1}=Q f_{1} T^{2} \\
& P_{2}=f_{1} T^{2} \sum_{i=2}^{p-1}\left(T^{2}-2 f_{i}\right) Q_{i}-Q f_{1} f_{p} .
\end{aligned}
$$


Set $C=R(\sqrt{-1})$ and let $V^{\prime}(C)$ (resp. $\tilde{W}(C)$, etc.) be the algebraic set over $C$ defined by $\mathfrak{p}^{e}=\mathfrak{p} C[T, \underline{X}]$. We define

$$
\begin{aligned}
& Z=\left\{(\underline{x}, t) \in C^{n+1}: P_{0}(\underline{x}, t)=P_{1}(\underline{x}, t)=P_{2}(\underline{x}, t)=0\right\}, \\
& H=\operatorname{Sing}\left(V^{\prime}(C)\right) \cup\left(Z \cap V^{\prime}(C)\right) .
\end{aligned}
$$

Then consider the mapping

$$
\phi: V^{\prime}(C) \backslash H \rightarrow \mathbf{P}_{2}(C)
$$

defined by $\phi(\underline{x}, t)=\left(P_{0}(\underline{x}, t), P_{1}(\underline{x}, t), P_{2}(\underline{x}, t)\right)$.

Let $\Lambda$ be the set of points $\left(\lambda_{1}, \lambda_{2}\right) \in C^{2}$ such that $\left\{P_{0}+\lambda_{1} P_{1}+\right.$ $\left.\lambda_{2} P_{2}=0\right\}$ is an irreducible and non-singular subvariety of $V^{\prime}(C) \backslash H$. Bertini's theorem (cf. $[\mathbf{H}]$, p. 275) assures that $\Lambda$ contains a Zariski open subset of $C^{2}$ provided that $\operatorname{dim}(\operatorname{im} \phi)=2$. Since $\Lambda^{\prime}=\left\{\left(\lambda_{1}, \lambda_{2}\right) \in R^{2}\right.$ : $\left.0<\lambda_{2}<\lambda_{1}\right\}$ is Zariski-dense in $C^{2}$, it is clear that we can take $\left(\lambda_{1}, \lambda_{2}\right)$ $\in \Lambda^{\prime} \cap \Lambda$ once we check the condition on the dimension of $\operatorname{im} \phi$. For that it is enough to check that there is no homogeneous polynomial $H\left(Y_{0}, Y_{1}, Y_{2}\right) \in C\left[Y_{0}, Y_{1}, Y_{2}\right]-\{0\}$ such that $H\left(P_{0}, P_{1}, P_{2}\right) \equiv 0\left(\bmod p^{e}\right)$.

Suppose the opposite and assume that $H$ is of degree $d$. Then

$$
H\left(Y_{0}, Y_{1}, Y_{2}\right)=\sum_{a+b+c=d} \alpha_{a b c} Y_{0}^{a} Y_{1}^{b} Y_{2}^{c} .
$$

Now, notice that since $\mathfrak{p}^{e}$ is generated by polynomials in $X_{1}, \ldots, X_{n}$ (but not in $T$ ), the equation

$$
A_{0}\left(X_{1}, \ldots, X_{n}\right)+A_{1}\left(X_{1}, \ldots, X_{n}\right) T+\cdots+A_{s}\left(X_{1}, \ldots, X_{n}\right) T^{2} \equiv 0
$$

$\left(\bmod \mathfrak{p}^{e}\right)$

implies $A_{i}\left(X_{1}, \ldots, X_{n}\right) \in \mathfrak{p}^{e}$ for all $i=0, \ldots, s$.

Thus we shall work on the lowest degree in $T$ of the monomials $P_{0}^{a} P_{1}^{b} P_{2}^{c}$. From (2.1.2) we get:

$$
\begin{aligned}
P_{0}^{a} P_{1}^{b} P_{2}^{c}= & \left(\prod_{i=2}^{p-1}\left(-f_{i}\right)\right)^{d}(-1)^{c} f_{1}^{b+c} f_{p}^{a+c} T^{2(a+b)} \\
& +T^{2(a+b)+1} G(X, T)
\end{aligned}
$$

(where in the case $p=2$ the first product is taken to be 1 ).

We will prove that $\alpha_{a b c}=0$ for all $a, b, c$. Set $h=a+b$. We work by induction on $h$. If $h=0$ then $a=b=0$ and we have to prove $\alpha_{00 d}=0$. Since the independent term of $H\left(P_{0}, P_{1}, P_{2}\right)$ is $\alpha_{00 d}\left(\prod_{i=1}^{p} f_{i}\right)^{d}$ we get $\alpha_{00 d}\left(\Pi_{i=1}^{d} f_{i}\right) \in \mathfrak{p}^{e}$. As $f_{i} \notin \mathfrak{p}^{e}, i=1, \ldots, p$, it is $\alpha_{00 d}=0$. 
Suppose $\alpha_{a^{\prime} b^{\prime} c^{\prime}}=0$ whenever $a^{\prime}+b^{\prime}<h$. Then

$$
H\left(P_{0}, P_{1}, P_{2}\right)=\sum_{\substack{a+b+c=d \\ a+b \geq h}} \alpha_{a b c} P_{0}^{a} P_{1}^{b} P_{2}^{c}=T^{2 h} M(T, X) .
$$

Since we have seen that $P_{0}^{a} P_{1}^{b} P_{2}^{c}=T^{2(a+b)} R(T, X)$, the term of degree $2 h$ in $H\left(P_{0}, P_{1}, P_{2}\right)$ comes from those $a, b, c$ such that $a+b=h$ and its coefficient is, after (2.1.3)

$$
\sum_{\substack{a+b+c=d \\ a+b=h}} \alpha_{a b c}(-1)^{d}\left(\prod_{i=2}^{p-1} f_{i}\right)^{d}(-1)^{c} f_{1}^{b+c} f_{p}^{a+c} .
$$

Thus we obtain

$$
\sum_{i=0}^{h} \alpha_{i, h-i, d-h} f_{1}^{d-i} f_{p}^{d-h+i} \equiv 0 \quad\left(\bmod \mathfrak{p}^{e}\right),
$$

which implies

$$
\sum_{i=0}^{h} \alpha_{i, h-i, d-h}\left(\frac{f_{p}}{f_{1}}\right)^{i}=0
$$

where this equation takes place in the function field of $V(C)$. But this means that $f_{p} / f_{1}$ is algebraic over $C$, that is, $f_{p}=\lambda f_{1}\left(\bmod \mathfrak{p}^{e}\right)$ for some $\lambda \in C$. Since $f_{1}, f_{p} \in R\left[X_{1}, \ldots, X_{n}\right]$, if we set $\lambda=\delta+\mu \sqrt{-1}, \delta, \mu \in R$, it follows $f_{p}=\delta f_{1} \bmod \left(\mathfrak{p}^{e} \cap R[X]\right)$.

As $\mathfrak{p}$ is real we get $\mathfrak{p}=\mathfrak{p}^{e} \cap R[X]$ and therefore $f_{p}=\delta f_{1}(\bmod \mathfrak{p})$, against our hypothesis on the choice of $f_{1}, \ldots, f_{p}$.

To complete the proof of Proposition 2.1 we shall show that

$$
\tilde{W}(C)=\left\{P_{0}+\lambda_{1} P_{1}+\lambda_{2} P_{2}=0\right\}
$$

is irreducible not only as a subvariety of $V^{\prime}(C) \backslash H$ but also as a subvariety of $V^{\prime}(C)$. For suppose this is done. Then if $\tilde{q}$ is the ideal of $\tilde{W}(C)$ in the coordinate ring of $V^{\prime}(C)$ we have that $q=\tilde{q} \cap R[V]$ is a prime ideal. On the other hand, since $\pi(W)=S$, where $W=\tilde{W}(C) \cap$ $R^{n+1}$ we get:

$$
\operatorname{dim} W=\operatorname{dim} \tilde{W}(C)=\operatorname{dim} V^{\prime}(C)-1
$$

which shows that $q$ is a real ideal. Therefore $W$ is an irreducible subvariety of $V^{\prime}$ in the conditions of 2.1 .

Finally, to prove that $\tilde{W}(C)$ is an irreducible subvariety of $V^{\prime}(C)$ we claim it is enough to prove that $\operatorname{codim} \pi(H) \geq 1$. Indeed, assume this is proved. Then, $\tilde{W}(C) \cap H$ has codimension $\geq 2$ in $V^{\prime}(C)$ since the morphism $\pi: \tilde{W}(C) \cap V^{\prime}(C) \rightarrow V(C)$ is finite (the polynomial $P_{0}+\lambda_{1} P_{1}+$ $\lambda_{2} P_{2} \in C[\underline{X}, T]$ is monic) and therefore has finite fibers on all the points of $H$. Thus, $\tilde{W}(C)$ has no components contained in $H$ and 2.1 is proved. 
Therefore the proposition follows at once from the lemma:

2.2. LemMA. Let $\pi: V^{\prime}(C) \rightarrow V(C)$ and $H=\operatorname{Sing}\left(V^{\prime}(C)\right) \cup(Z \cap$ $\left.V^{\prime}(C)\right)$ be as above. Then, $\operatorname{codim} \pi(H) \geq 1$.

Proof. Since $V^{\prime}(C)=V(C) \times C$ it is clear that $\operatorname{codim}\left(\operatorname{Sing}\left(V^{\prime}(C)\right)\right)$ $\geq 1$. Thus it is enough to show $\operatorname{codim} \pi\left(Z \cap V^{\prime}(C)\right) \geq 1$. Let $\underline{a} \in$ $\pi\left(Z \cap V^{\prime}(C)\right)$. Then there is $t \in C$ such that $P_{0}(\underline{a}, t)=P_{1}(\underline{a}, t)=$ $P_{2}(\underline{a}, t)=0$. From $P_{1}(\underline{a}, t)=0$ we get one of the following possibilities (check (2.1.2)):

(i) $f_{1}(\underline{a})=0$. Then $\underline{a} \in V(C) \cap\left\{f_{1}=0\right\}$.

(ii) $t=0$. But then from $P_{2}(\underline{a}, 0)=0$ it follows readily $\underline{a} \in V(C) \cap$ $\left\{\Pi_{i=1}^{p} f_{i}=0\right\}$.

(iii) $Q(\underline{a}, t)=0$. Then, from $P_{0}(\underline{a}, t)=0$ we get also

$$
t^{4} \sum_{i=2}^{p-1}\left(t^{2}-2 f_{i}(\underline{a})\right) Q_{i}(\underline{a}, t)=0 .
$$

If $t=0$ by (ii) it is $a \in\left\{\prod_{i=1}^{p} f_{i}=0\right\}$. Thus we are reduced to the system

$$
\left\{\begin{array}{l}
Q(\underline{a}, t)=0 \\
\sum_{i=2}^{p-1}\left(t^{2}-2 f_{i}(\underline{a})\right) Q_{i}(\underline{a}, t)=0 .
\end{array}\right.
$$

As $Q(\underline{a}, t)=\prod_{i=2}^{p-1}\left(t^{2}-f_{i}(\underline{a})\right)($ see $(2.1 .1))$ we have $t^{2}-f_{j}(\underline{a})=0$ for some $j$. If there are $i, j, i \neq j$, with $t^{2}-f_{i}(\underline{a})=t^{2}-f_{j}(\underline{a})=0$ it follows $\underline{a} \in V(C) \cap\left\{f_{i}-f_{j}=0\right\}$. Now assume $t^{2}-f_{j}(\underline{a})=0$ only for one $j$, say $j=2$. From the very definition of $Q_{i}$ (see (2.1.1)), the second equation of (2.2.1) reduces to

$$
\left(t^{2}-2 f_{2}(\underline{a})\right) Q_{2}(\underline{a}, t)=0 .
$$

Again, either $\underline{a} \in V(C) \cap\left\{f_{2}=0\right\}$, or

$$
Q_{2}(\underline{a}, t)=\prod_{j>2}^{p-1}\left(t^{2}-f_{j}(\underline{a})\right)=0
$$

whence $\underline{a} \in V(C) \cap\left\{f_{2}-f_{j}=0\right\}$ for some $j>2$.

In conclusion, in any case we have

$$
\underline{a} \in V(C) \cap\left\{\left(\prod_{i=1}^{p} f_{i}\right)\left(\prod_{i<j}\left(f_{i}-f_{j}\right)\right)=0\right\}
$$


and since by hypothesis $f_{i} \notin \mathfrak{p}$ and $f_{i}-f_{j} \notin \mathfrak{p}$ all $i, j$, we have

$$
\operatorname{codim} \pi(Z \cap V(C)) \geq 1
$$

and the proof is complete.

2.3. REmark. Note that if $p=1$ in Proposition 2.1 , the above proof works by setting $f_{2}=-1$.

3. The main result. We start with the following:

3.1. Definition. A semialgebraic set $S \subset R^{n}$ is called irreducible if the smallest algebraic set which contains $S$ is irreducible (i.e. $\mathscr{J}(S)=\left\{f \in R\left[X_{1}, \ldots, X_{n}\right]: f(x)=0\right.$ all $\left.x \in S\right\}$ is a prime ideal of $\left.R\left[X_{1}, \ldots, X_{n}\right]\right)$.

Now we are ready to prove the main result:

3.2. TheOREM. Let $S \subset R^{n}$ be an irreducible closed semialgebraic subset. Let $V \subset R^{n}$ be the Zariski closure of $S$. Then there exist a positive integer $m$ and an irreducible algebraic set $W \subset R^{n+m}$ such that:

(1) $\pi: W \rightarrow V$ is finite.

(2) $\pi(W)=S$.

Proof. We may assume $S$ written in the form (see [A-G])

$$
S=\left(S_{1} \cap \cdots \cap S_{m}\right) \cap V
$$

with

$$
S_{i}=\left\{f_{1 i} \geq 0\right\} \cup \cdots \cup\left\{f_{p i} \geq 0\right\},
$$

where for each $(k, i) \in\{1, \ldots, p\} \times\{1, \ldots, m\} \quad f_{k i} \in R\left[X_{1}, \ldots, X_{n}\right]$. Then we will find $W \subset R^{n+m}$. We work by induction on $m$.

For $m=1$ the theorem is Proposition 2.1. Assume now that there exists an irreducible algebraic set $X^{\prime} \subset R^{n+m-1}$ such that

(i) $\pi\left(X^{\prime}\right) \rightarrow V$ is finite, and

(ii) $\pi\left(X^{\prime}\right)=S^{\prime}=\left(S_{1} \cap \cdots \cap S_{m-1}\right) \cap V$.

(Notice that $V$ is still the Zariski closure of $S^{\prime}$.) Let $X=X^{\prime} \times R \subset R^{n+m}$ be the algebraic set defined by the extension of the ideal of $X^{\prime}, \mathscr{J}\left(X^{\prime}\right) \subset$ $R\left[X_{1}, \ldots, X_{n}, T_{1}, \ldots, T_{m-1}\right]$, to the polynomial $\operatorname{ring} R\left[X_{1}, \ldots, X_{n}\right.$, $\left.T_{1}, \ldots, T_{m-1}, T\right]$ where $T$ is a new variable. Obviously $X$ is irreducible and $\pi(X)=S^{\prime}$. 
Now let $F(T, \underline{X})=P_{0}+\lambda_{1} P_{1}+\lambda_{2} P_{2} \in R\left[X_{1}, \ldots, X_{n}, T\right]$ be the polynomial displayed in 2.1.2 with the property that for any $\lambda_{1}, \lambda_{2} \in R$, $0<\lambda_{2}<\lambda_{1}$, the set $Y^{\prime}$ of zeros of $F$ (as a subset of $R^{n+1}$ ) projects onto $S_{m}$. Let $Y$ be the algebraic subset of $R^{n+m}$ defined by $F(T, \underline{X})$ considered as a polynomial in $R\left[X_{1}, \ldots, X_{n}, T_{1}, \ldots, T_{m-1}, T\right]$. Then $\pi:(X \cap Y) \rightarrow V$ is finite since both $\pi: X^{\prime} \rightarrow V$ and $\pi: Y^{\prime} \rightarrow R^{n}$ are finite, and $\pi(X \cap Y)$ $=S$.

Let $C=R(\sqrt{-1})$ be the algebraic closure of $R$ and consider

$$
Z=\left\{\left(\underline{x}, t_{1}, \ldots, t_{m-1}, t\right) \in Y(C): P_{0}(\underline{x}, t)=P_{1}(\underline{x}, t)=P_{2}(\underline{x}, t)=0\right\} \text {. }
$$

After Lemma 2.2 it is $\operatorname{codim}(\pi(Z)) \geq 1$. Let $H=\operatorname{Sing}(X(C)) \cup(Z \cap$ $X(C))$. Then $\operatorname{codim}(\pi(H)) \geq 1$ since we have $\operatorname{dim} X^{\prime}=\operatorname{dim} V$. Consider

$$
\phi: X(C) \backslash H \rightarrow P_{2}(C)
$$

defined by

$$
\phi\left(\underline{x}, t_{1}, \ldots, t_{m-1}, t\right)=\left(P_{0}(\underline{x}, t), P_{1}(\underline{x}, t), P_{2}(\underline{x}, t)\right) .
$$

Since $X(C) \backslash H$ is non-singular, Bertini's theorem applies assuring that the set of points $\left(\lambda_{1}, \lambda_{2}\right) \in C^{2}$ such that $(X(C) \backslash H) \cap Y(C)$ is an irreducible, non-singular subvariety of $X(C) \backslash H$, contains a Zariski open subset of $C^{2}$ provided that $\operatorname{dim}(\operatorname{im} \phi)=2$. Since $R$ is Zariski-dense in $C$ we can choose $\lambda_{1}, \lambda_{2} \in R, 0<\lambda_{2}<\lambda_{1}$.

As $\pi(X)=S^{\prime}$ which is Zariski-dense in $V$, to prove that $\operatorname{dim}(\operatorname{im} \phi)=$ 2 it is enough that $P_{0}, P_{1}$ and $P_{2}$ do not verify any homogeneous polynomial, modulo $\mathfrak{p}^{e}=\mathscr{J}(V) C[\underline{X}]$ and this was shown in the proof of Proposition 2.1. Finally, since $\operatorname{codim}(\pi(H)) \geq 1$ it follows that $Y(C) \cap$ $X(C)$ is irreducible in $X(C)$. Thus, as in 2.1 the real algebraic set $W=Y \cap X$ verifies the conditions of the theorem.

3.3. Definition. Let $S$ be a semialgebraic subset of $R^{n}$ and let $V$ be its Zariski closure. We say that $S$ is regularly closed in $V$ if it coincides with the closure of its interior, where both, closure and interior are taken in the order topology of $V$ (also called strong topology).

3.4. Corollary. Let $S, V$ and $W$ be as in Theorem 3.2. Assume further that $S$ is regularly closed in $V$. Then $\pi\left(W_{c}\right)=S$.

Proof. Since $\pi: W \rightarrow V$ is finite, $\pi\left(W_{c}\right)$ is a closed semialgebraic subset of $V$ (see [B] p. 170). So it is enough to show that $\dot{S} \subset \pi\left(W_{c}\right)$.

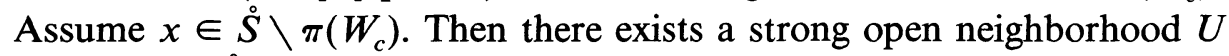
of $x, U \subset \stackrel{S}{S}$, such that $U \cap \pi\left(W_{c}\right)=\varnothing$. Thus $U \subset \pi\left(W \backslash W_{c}\right)$. But $\operatorname{dim}\left(\pi\left(W \backslash W_{c}\right)\right)<\operatorname{dim} W=\operatorname{dim} V=\operatorname{dim} U$ (once more by the finiteness of $\pi)$, contradiction. 
3.5. REMARK. Notice that the condition of the irreducibility of $S$ in Theorem 3.2 is necessary. Indeed it is immediate to check that no irreducible real algebraic set can project onto the semialgebraic consisting of two points.

4. Applications. We describe first of all the applications of Theorems 3.2 to the order space of a function field. For that let $K=R\left(x_{1}, \ldots, x_{n}\right)$ be a real function field of transcendence degree $r$ over $R$. We denote by $X(K)$ its space of orders endowed with Harrison's topology. Given any formally real extension $E$ of $K$ we consider the restriction morphism $\varepsilon$ defined by

$$
\varepsilon: X(E) \rightarrow X(K): P \rightarrow P \cap K .
$$

A subset $Y$ of $X(K)$ is called clopen if it is open and closed in the Harrison's topology of $X(K)$ (cf. [E-L-W]). Since $X(K)$ is compact ([P]), we can write $Y$ as a finite union of basic open sets

$$
Y=H_{1} \cup \cdots \cup H_{p},
$$

where

$$
H_{i}=H\left(f_{1 i}, \ldots, f_{r i}\right)=\left\{P \in X(K): f_{1 i} \in P, \ldots, f_{r i} \in P\right\}
$$

and all the $f_{k i}$ are in any ring $A$ with quotient field $K$.

4.1. THEOREM. Let $Y$ be any clopen subset of $X(K)$. Then there exists a finite extension $E$ of $K$ such that $Y=\operatorname{im} \varepsilon$.

Proof. Let $V$ be a non-singular model of $K$ and assume that $R\left[x_{1}, \ldots, x_{n}\right]$ is the coordinate ring of $V$. We write $Y=H_{1} \cup \cdots \cup H_{p}$ with $H_{i}=H\left(f_{1 i}, \ldots, f_{r i}\right), f_{k i} \in R\left[x_{1}, \ldots, x_{n}\right]$. We define the semialgebraic set associated to $Y$ by

$$
\hat{Y=} \hat{H_{1}} \cup \cdots \cup \hat{H_{p}}
$$

where

$$
H_{i}^{\hat{}}=\left\{x \in V: f_{1 i}(x)>0, \ldots, f_{r i}(x)>0\right\} .
$$

Let $\bar{Y}^{\wedge}$ be the strong closure of $Y^{\wedge}$ in $V$. Then $\bar{Y}^{\wedge}$ is a semialgebraic set, regularly closed in $V$. Then by 3.4 there exists an irreducible algebraic set $W \subset R^{n+m}$ such that $\pi(W)=\pi\left(W_{c}\right)=\bar{Y}$. Let $E$ be the function field of $W$. Since $\pi$ is finite $E$ is a finite extension of $K$. Furthermore, from $\pi\left(W_{c}\right)=\bar{Y}^{\wedge}$ it follows (see [D-R], Prop. 2.10) that $Y=\operatorname{im} \varepsilon$, what achieves the proof. 
Theorem 3.2 can also be stated in terms of the real spectrum of real finitely generated $R$-algebras as follows (we refer to $[\mathbf{C}-\mathbf{R}]$ for the definition and main properties of the real spectrum).

4.2. Corollary. Let $A$ be a real domain, finitely generated as $R$-algebra. Let $C$ be a closed constructible set of $\operatorname{Spec}_{R} A$ with non-empty interior. Then there exists a real domain $B$, integral over $A$ such that $C=j^{*}\left(\operatorname{Spec}_{R} B\right)$.

Proof. In [C-R] it is proved that if $V, W$ are algebraic sets and $f$ : $V \rightarrow W$ is a morphism, then the diagram

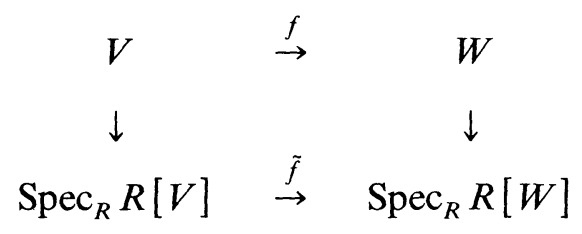

is commutative, where the vertical arrows are the natural inclusions of $V$ and $W$ into their real spectra (see $[\mathbf{C}-\mathbf{R}]$ ). On the other hand in $[\mathbf{C}-\mathbf{R}]$ it is proved that the correspondence between closed constructible sets $\operatorname{Spec}_{R} R[V]$ and closed semialgebraic sets of $V$ via the inclusion $V \rightarrow$ $\operatorname{Spec}_{R} R[V]$ is one to one. Then 4.2 follows at once from Theorem 3.2.

We end the paragraph showing how Theorem 3.2 can be used to produce "exotic" real irreducible algebraic sets. For it we define a semialgebraic set to be connected if it is not the union of two disjoint and non-empty open semialgebraic sets (see [B]).

Then we show:

4.3. Proposition. For any natural numbers $n, p$, there exists an irreducible, non-singular real hypersurface in $\mathbf{P}^{n+1}(R)$ with $p$ equidimensional connected components.

Proof. Clearly it is enough to find a non-singular, irreducible, bounded affine hypersurface of $R^{n+1}$ verifying the requested conditions. The case $p=1$ is, obviously, trivial.

To get hypersurfaces with $p$ connected components, we do the following: we take $p$ disjoint balls, $B_{1}, \ldots, B_{p}$, with

$$
B_{j}=\left\{x \in R^{n}: f_{j}(x) \geq 0\right\}, \quad f_{j}(X)=r_{j}^{2}-\sum_{i=1}^{n}\left(X_{i}-a_{j l}\right)^{2} .
$$


Then we use Proposition 2.1 (for $V=R^{n}$ ) to produce an irreducible hypersurface $W \subset R^{n+1}$, of equation $F(T, X)=0$, which projects onto $S=B_{1} \cup \cdots \cup B_{p}$. It is clear that $W$ has at least $p$ connected components. Now it is a matter of working out the equation $F$ and playing with the centers $\underline{a}_{j}$ and the radius $r_{j}$ of the balls $B_{j}$ to show that $W$ has indeed only $p$ connected components (therefore of dimension $n$ ) and is non-singular.

Let $s_{j}\left(x_{1}, \ldots, x_{l}\right)$ denote the $j$ th elementary symmetric function. Then the polynomial $F(T, X)$ of 2.1 takes the form

$$
\begin{aligned}
F(T, X) P= & (p-1) T^{2 p} \\
& +\sum_{j=1}^{p-1} M_{j}(X) T^{2(p-j)}+(-1)^{p} \lambda_{2} f_{1} \cdots f_{p}
\end{aligned}
$$

where

$$
\begin{aligned}
(-1)^{j} M_{j}(X)= & s_{j}\left(\lambda_{1} f_{1}, \ldots, f_{p-1}\right)+f_{p} s_{J-1}\left(\lambda_{2} f_{1}, \ldots, f_{p-1}\right) \\
& +\sum_{i=2}^{p-1} s_{J}\left(\lambda_{2} f_{1}, \ldots, 2 f_{l}, \ldots, f_{p-1}\right) .
\end{aligned}
$$

Now, taking into account that if $f_{k}(x)>0$ for some $k$ then $f_{l}(x)<0$ for all $l \neq k$ one can check, by working on $M_{j}(x)$ that we can choose $\underline{a}_{j} \in R^{n}$ and $r_{j} \in R, j=1, \ldots, p$, such that for all $x \in S$ it is $M_{j}(x)>0$, $j=1, \ldots, p$. Thus it is immediate from 4.3.1 that over any $x \in S$ we have either two points if $\Pi_{k=1}^{p} f_{k}(x) \neq 0$, or a unique point, $(x, 0)$, in case $f_{k}(x)=0$ for some $k=1, \ldots, p$. Since $\pi: W \rightarrow R^{n}$ is finite this implies that $W$ has exactly one connected component over each ball $B_{1}, \ldots, B_{p}$.

That $F(T, X)$ is non-singular follows readily by taking derivatives in (4.3.1). We have

$$
\frac{\partial F}{\partial T}=T\left(2 p(p-1) T^{2(p-1)}+\sum_{j=1}^{p-1} 2(p-j) M_{j}(x) T^{2(p-j-1)}\right) .
$$

Since $M_{j}(x)>0$ for $x \in \pi(V),(\partial F / \partial T)(x, t)=0$ implies $t=0$. But $(x, 0) \in \operatorname{Sing}(V)$ implies $x$ is a singular point of some of the spheres $\left\{f_{j}=0\right\}, j=1, \ldots, p$, absurd.

Concerning the dimension anomalies of real algebraic sets we prove:

4.4. Proposition. For any finite increasing sequence of natural numbers $s_{0}<s_{1}<\cdots<s_{r}$ there exists an irreducible real algebraic set of dimension $s_{r}$ with pieces of dimensions $s_{0}, s_{1}, \ldots, s_{r}$ (and no pieces of other dimensions). 
Proof. In $R^{s_{r}}$ we consider linear subspaces $L_{k}$ of dimension $s_{k}$, transversal each other, for $k=0, \ldots, r-1$. Then, if $L_{k}$ is defined by the linear forms $l_{1}, \ldots, l_{s_{r}-s_{k}}$ we have $L_{k}=\left\{f_{k} \geq 0\right\}$ where $f_{k}=$ $-\left(l_{1}^{2}+\cdots+l_{s_{r}-s_{k}}^{2}\right)$. Now let $B$ be a closed ball in $R^{s_{r}}$ of radius $\varepsilon$ and center at a point $\underline{a} \in R^{s_{r}}$ with $\|\underline{a}\|$ large enough, tangent to the linear space $L_{s_{r-1}}$ and such that $L_{s_{k}} \cap B=\varnothing$ for all $k=0, \ldots, r-2$. Notice that $B=\left\{f_{r} \geq 0\right\}$, where $f_{r}=\left[\varepsilon-\sum_{i=1}^{s_{r}}\left(x_{i}-a_{i}\right)^{2}\right]^{t}, t$ odd. Thus by taking $\varepsilon$ small enough and $t$ suitably large we can make the positive values of $f_{r}$ as small as needed. Then consider the semialgebraic set

$$
S=\left\{f_{0} \geq 0\right\} \cup \cdots \cup\left\{f_{r-1} \geq 0\right\} \cup\left\{f_{r} \geq 0\right\} .
$$

Let $W$ be the hypersurface produced by Proposition 2.1 which projects onto $S$. Writing the equation of $W$ as in the proof of 4.3 it is immediate, from the fact that $f_{k} \leq 0$ over $R^{s_{r}}$ for all $k=0, \ldots, r-1$, and the remark on $f_{r}$ made above that we can choose $\varepsilon, \underline{a}$ and $t$ such that it is $M_{j}(x)>0$ for all $x \in S$ and all $j$. Thus, as in 4.3, $W$ consists of one single point over all the linear subspaces (hence coincides with them) and two (symmetric) points over $B$ which coincide over the boundary of $B$. Hence 4.4 follows at once.

4.5. Remark. Notice that from the proof of 4.4 it follows that $W$ is connected when $s_{0} \geq 1$.

\section{REFERENCES}

[A-G] C. Andradas and J. M. Gamboa, A note on projections of real algebraic sets, Pacific J. Math., 115 (1984), 1-11.

[B] G. W. Brumfiel, Partially ordered fields and semialgebraic geometry, London Math. Soc. Lecture Notes 37, (1979).

[C-R] M. Coste and M. F. Roy, La topologie du spectre réel, Cont. Math., 8 (1982), 27-59.

[D-R] D. W. Dubois and T. Recio, Order extensions and real algebraic geometry, Cont. Math., 8 (1982), 265-288.

[E-L-W] R. Elman, T. Y. Lam and A. Wadsworth, Orderings under field extensions, J. reine ang. Math., 306 (1979), 7-27.

[H] R. Hartshorne, Algebraic Geometry, G.T.M. 52, Springer-Verlag, (1977).

[P] A. Prestel, Lectures on formally real fields, I.M.P.A. no. 25, (1975).

Received June 7, 1984 and in revised form October 22, 1984. Partially supported by CAICYT no. $2280 / 83$. 



\title{
PACIFIC JOURNAL OF MATHEMATICS EDITORS
}

V. S. VARADARAJAN (Managing Editor)
University of California
Los Angeles, CA 90024
HEBERT ClEMENS
University of Utah
Salt Lake City, UT 84112
CHARLES R. DEPRIMA
California Institute of Technology
Pasadena, CA 91125

R. FINN

Stanford University

Stanford, CA 94305

HERMANN FLASCHKA

University of Arizona

Tucson, AZ 85721

Ramesh A. Gangolli

University of Washington

Seattle, WA 98195

ROBION KIRBY

University of California

Berkeley, CA 94720
C. C. MOORE

University of California

Berkeley, CA 94720

H. SAMELSON

Stanford University

Stanford, CA 94305

HaRold Stark

University of California, San Diego

La Jolla, CA 92093

\section{ASSOCIATE EDITORS}

\author{
R. ARENS \\ E. F. BECKENBACH \\ B. H. NeumanN \\ F. Wolf \\ K. YoSHIDA \\ (1906-1982)
}

\section{SUPPORTING INSTITUTIONS}

UNIVERSITY OF ARIZONA
UNIVERSITY OF BRITISH COLUMBIA
CALIFORNIA INSTITUTE OF TECHNOLOGY
UNIVERSITY OF CALIFORNIA
MONTANA STATE UNIVERSITY
UNIVERSITY OF NEVADA, RENO
NEW MEXICO STATE UNIVERSITY
OREGON STATE UNIVERSITY

UNIVERSITY OF OREGON

UNIVERSITY OF SOUTHERN CALIFORNIA

STANFORD UNIVERSITY

UNIVERSITY OF HAWAII

UNIVERSITY OF TOKYO

UNIVERSITY OF UTAH

WASHINGTON STATE UNIVERSITY UNIVERSITY OF WASHINGTON

The Supporting Institutions listed above contribute to the cost of publication of this Journal, but they are not owners or publishers and have no responsibility for its content or policies.

Mathematical papers intended for publication in the Pacific Journal of Mathematics should be in typed form or offset-reproduced (not dittoed), double spaced with large margins. Please do not use built up fractions in the text of the manuscript. However, you may use them in the displayed equations. Underline Greek letters in red, German in green, and script in blue. The first paragraph must be capable of being used separately as a synopsis of the entire paper. In particular it should contain no bibliographic references. Please propose a heading for the odd numbered pages of less than 35 characters. Manuscripts, in triplicate, may be sent to any one of the editors. Please classify according to the scheme of Math. Reviews, Index to Vol. 39. Supply name and address of author to whom proofs should be sent. All other communications should be addressed to the managing editor, or Elaine Barth, University of California, Los Angeles, California 90024.

There are page-charges associated with articles appearing in the Pacific Journal of Mathematics. These charges are expected to be paid by the author's University, Government Agency or Company. If the author or authors do not have access to such Institutional support these charges are waived. Single authors will receive 50 free reprints; joint authors will receive a total of 100 free reprints. Additional copies may be obtained at cost in multiples of 50 .

The Pacific Journal of Mathematics is issued monthly as of January 1966. Regular subscription rate: $\$ 190.00$ a year (5 Vols., 10 issues). Special rate: $\$ 95.00$ a year to individual members of supporting institutions.

Subscriptions, orders for numbers issued in the last three calendar years, and changes of address should be sent to Pacific Journal of Mathematics, P.O. Box 969, Carmel Valley, CA 93924, U.S.A. Old back numbers obtainable from Kraus Periodicals Co., Route 100, Millwood, NY 10546.

The Pacific Journal of Mathematics at P.O. Box 969, Carmel Valley, CA 93924 (ISSN 0030-8730) publishes 5 volumes per year. Application to mail at Second-class postage rates is pending at Carmel Valley, California, and additional mailing offices. Postmaster: Send address changes to Pacific Journal of Mathematics, P.O. Box 969, Carmel Valley, CA 93924.

\section{PUBLISHED BY PACIFIC JOURNAL OF MATHEMATICS, A NON-PROFIT CORPORATION}

Copyright $\subset 1986$ by Pacific Journal of Mathematics 


\section{Pacific Journal of Mathematics}

\section{Vol. 121, No. 2 December, 1986}

Jorge Almeida, Minimal nonpermutative pseudovarieties of semigroups.

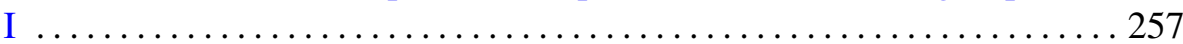

Jorge Almeida, Minimal nonpermutative pseudovarieties of semigroups.

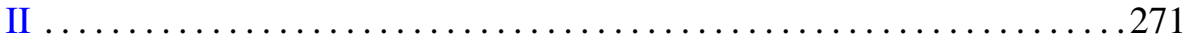

Carlos Andradas Heranz and José Manuel Gamboa Mutuberría, On

projections of real algebraic varieties $\ldots \ldots \ldots \ldots \ldots \ldots \ldots \ldots \ldots 281$

Zeev Ditzian, Inverse theorems for multidimensional Bernstein operators . . 293

M. Furi and Maria Patrizia Pera, A continuation principle for forced oscillations on differentiable manifolds $\ldots \ldots \ldots \ldots \ldots \ldots \ldots \ldots \ldots . \ldots . \ldots . \ldots 321$

James J. Hebda, The collars of a Riemannian manifold and stable

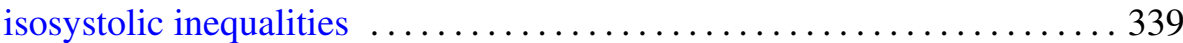

Henryk Hecht and Dragan Miličić, Character identities and asymptotic behavior of matrix coefficients of discrete series ................ 357

Piotr Jakóbczak, The boundary regularity of the solution of the $\bar{\partial}$-equation in the product of strictly pseudoconvex domains $\ldots \ldots \ldots \ldots \ldots \ldots . \ldots 371$

Krzysztof Jarosz, Isometries between injective tensor products of Banach

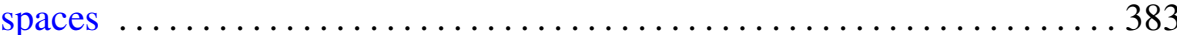

Hans Keller, On valued, complete fields and their automorphisms ........ 397

David Masser and Peter Man-Kit Shiu, On sparsely totient numbers . . . . 407

Tze-Beng Ng, Vector bundles over $(8 k+3)$-dimensional manifolds

Thomas Joseph Ransford, The spectrum of an interpolated operator and analytic multivalued functions

Akihito Uchiyama, On the radial maximal function of distributions 467 Jang-Mei Gloria Wu, On singularity of harmonic measure in space 